UdeM-GPP-TH-02-105

\title{
$B_{d, s}^{0} \rightarrow K^{(*)} \bar{K}^{(*)}:$ CP phase $\alpha$ and New Physics 由
}

\author{
Alakabha Datta and David London \\ Laboratoire René J.-A. Lévesque, Université de Montréal, \\ C.P. 6128, succ. centre-ville, Montréal, QC, Canada H3C 3J7
}

(December 24, 2018)

\begin{abstract}
The decays $B_{d, s}^{0} \rightarrow K^{(*)} \bar{K}^{(*)}$ can be used to measure the angle $\alpha$ of the CKM unitarity triangle. The theoretical error from $S U(3)$ breaking is expected to be small, so that the determination of $\alpha$ is clean. Moreover, since $B_{d, s}^{0} \rightarrow K^{(*)} \bar{K}^{(*)}$ are pure penguin decays, they are particularly sensitive to the presence of new physics.
\end{abstract}

\footnotetext{
${ }^{1}$ Talk presented by Alakabha Datta at the 5th International Conference on Hyperon, Charm and Beauty Hadrons.
} 


\section{$1 \quad$ CP phase $\alpha$ from $B_{d, s}^{0} \rightarrow K^{(*)} \bar{K}^{(*)}$}

The first evidence of $\mathrm{CP}$ violation in the $B$ system was recently observed with the measurement of one of the angles of the Cabibbo-Kobayashi-Maskawa (CKM) unitarity triangle: $\sin 2 \beta=0.78 \pm 0.08$ [1], which is consistent with the standard model (SM). Future efforts will focus on the measurement of the remaining two angles of the unitarity triangle, $\alpha$ and $\gamma$, in order to test the SM explanation of $\mathrm{CP}$ violation.

There are two standard techniques for the extraction of $\alpha$. The first method uses the CP asymmetry in $B_{d}^{0}(t) \rightarrow \pi^{+} \pi^{-}$to obtain $\alpha$. Unfortunately, there is a penguin contribution, making it necessary to perform an isospin analysis of $B \rightarrow \pi \pi$ decays [2]. This requires the measurement of $B_{d}^{0} \rightarrow \pi^{0} \pi^{0}$, which is expected to have a small branching ratio. Hence, it may be difficult to obtain $\alpha$ using this method. The second method uses a Dalitz-plot analysis of $B_{d}^{0}(t) \rightarrow \rho \pi \rightarrow \pi^{+} \pi^{-} \pi^{0}$ decays [3]. However, the unknown non-resonant background and the correct description of $\rho \rightarrow \pi \pi$ decays are factors that can seriously affect a clean determination of $\alpha$ using this method.

In this talk, we present a new method for determining $\alpha$ [4]. As a starting point, consider the pure $b \rightarrow d$ penguin decay $B_{d}^{0} \rightarrow K^{0} \bar{K}^{0}$, for which the underlying quark transition is $\bar{b} \rightarrow \bar{d} s \bar{s}$. The amplitude for $B_{d}^{0} \rightarrow K^{0} \bar{K}^{0}, A_{d}$, can be written as

$$
\begin{aligned}
A_{d} & =P_{u} V_{u}^{d}+P_{c} V_{c}^{d}+P_{t} V_{t}^{d} \\
& =\left(P_{u}-P_{c}\right) V_{u}^{d}+\left(P_{t}-P_{c}\right) V_{t}^{d}
\end{aligned}
$$

where $V_{q}^{d} \equiv V_{q b}^{*} V_{q d}$, and $P_{u, c, t}$ are the penguin amplitudes. In passing from the first line to the second, we have used the unitarity of the CKM matrix, $V_{u b}^{*} V_{u d}+V_{c b}^{*} V_{c d}+V_{t b}^{*} V_{t d}=0$, to eliminate the $V_{c b}^{*} V_{c d}$ term. The amplitude $\bar{A}_{d}$ describing the conjugate decay $\bar{B}_{d}^{0} \rightarrow K^{0} \bar{K}^{0}$ can be obtained from the above by changing the signs of the weak phases.

By making time-dependent measurements of $B_{d}^{0}(t) \rightarrow K^{0} \bar{K}^{0}$, one can obtain the three observables

$$
\begin{aligned}
X & \equiv \frac{1}{2}\left(\left|A_{d}\right|^{2}+\left|\bar{A}_{d}\right|^{2}\right) \\
Y & \equiv \frac{1}{2}\left(\left|A_{d}\right|^{2}-\left|\bar{A}_{d}\right|^{2}\right) \\
Z_{I} & \equiv \operatorname{Im}\left(e^{-2 i \beta} A_{d}^{*} \bar{A}_{d}\right) .
\end{aligned}
$$

The three independent observables depend on four theoretical parameters: $P_{u c} \equiv\left|P_{u}-P_{c}\right|$, $P_{t c} \equiv\left|P_{t}-P_{c}\right|$, the relative weak phase between the two amplitudes, $\alpha$, and the relative strong phase. Hence one cannot obtain CP phase information from these measurements [5]. However, substituting Eq. 1 in Eq. 2, one can obtain

$$
P_{t c}^{2}\left|V_{t}^{d}\right|^{2}=\frac{Z_{R} \cos 2 \alpha+Z_{I} \sin 2 \alpha-X}{\cos 2 \alpha-1},
$$


where

$$
Z_{R} \equiv \operatorname{Re}\left(e^{-2 i \beta} A_{d}^{*} \bar{A}_{d}\right)
$$

The quantity $Z_{R}$ is related to the three observables in Eq. 2 by

$$
Z_{R}^{2}=X^{2}-Y^{2}-Z_{I}^{2}
$$

Now consider a second pure $b \rightarrow d$ penguin decay of the form $B_{d}^{0} \rightarrow K^{*} \bar{K}^{*}$. Here $K^{*}$ represents the ground state vector meson, $K^{*}(892)$, or any excited neutral kaon, such as $K_{1}(1270)$, etc. This second decay can be treated in a similar fashion as the first one above, with unprimed parameters and observables being replaced by primed ones. One can then combine measurements of the two decays to obtain

$$
\begin{aligned}
r_{d} \equiv \frac{P_{t c}^{2}}{P_{t c^{\prime}}^{2}} & =\frac{Z_{I} \sin 2 \alpha+Z_{R} \cos 2 \alpha-X}{Z_{I}^{\prime} \sin 2 \alpha+Z_{R}^{\prime} \cos 2 \alpha-X^{\prime}} \\
& =f(\alpha) .
\end{aligned}
$$

The equation above, $r_{d}=f(\alpha)$, could then be solved for $\alpha$ if we knew $r_{d}$. Note that the CKM elements on the left-hand side of Eq. 3 cancel in constructing the ratio $r_{d}$.

Information about the ratio $r_{d}$ can be obtained by measuring $B_{s}^{0}$ decays to the same final states $K^{0} \bar{K}^{0}$ and $K^{*} \bar{K}^{*}$. Consider first the decay $B_{s}^{0} \rightarrow K^{0} \bar{K}^{0}$. This is described by a $b \rightarrow s$ penguin amplitude, $A_{s}$, which is given by

$$
\begin{aligned}
A_{s} & =P_{u}^{(s)} V_{u}^{s}+P_{c}^{(s)} V_{c}^{s}+P_{t}^{(s)} V_{t}^{s} \\
& \simeq\left(P_{t}^{(s)}-P_{c}^{(s)}\right) V_{t}^{s}
\end{aligned}
$$

where $V_{q}^{s} \equiv V_{q b}^{*} V_{q s}$, and $P_{u, c, t}^{s}$ are the penguin amplitudes. In writing the second line, we have again used the unitarity of the CKM matrix to eliminate the $V_{c b}^{*} V_{c s}$ piece. Furthermore, the $V_{u b}^{*} V_{u s}$ piece is negligible: $\left|V_{u b}^{*} V_{u s}\right| \ll\left|V_{t b}^{*} V_{t s}\right|$. Thus, the measurement of the branching ratio for $B_{s}^{0} \rightarrow K^{0} \bar{K}^{0}$ yields $\left|P_{t}^{s}-P_{c}^{s}\right|\left|V_{t}^{s}\right|$. Similarly one can obtain $\left|P_{t}^{\prime s}-P_{c}^{\prime} s\right|\left|V_{t}^{s}\right|$ from the branching ratio for $B_{s}^{0} \rightarrow K^{*} \bar{K}^{*}$. In this way, we can measure

$$
r_{s} \equiv \frac{P_{t c}^{(s)^{2}}}{P_{t c}^{\prime(s)^{2}}},
$$

where $P_{t c}^{s} \equiv\left|P_{t}^{s}-P_{c}^{s}\right|$ and $P_{t c}^{\prime s} \equiv\left|P_{t}^{\prime} s-P_{c}^{\prime} s\right|$.

Now, the main point is that, up to small $S U(3)$ corrections,

$$
r_{d}=r_{s}
$$

Note again that the CKM matrix elements cancel in both ratios. The $S U(3)$ breaking in the equality $r_{d}=r_{s}$ is at the level of $5 \%$ or less 4 . Hence $\alpha$ can be obtained from the equation $r_{d} \approx r_{s}=f(\alpha)$. This equation has several solutions and so there are discrete 
ambiguities in the extraction of $\alpha$. However, by comparing several pairs of processes, the discrete ambiguities can be eliminated. In fact, with one theoretical assumption, all the discrete ambiguities can be removed with a single pair of processes [4].

This method can also be used when the final state is not self-conjugate. For example, one can consider the decays $B_{d}^{0} \rightarrow K^{0} \bar{K}^{*}$ and $B_{d}^{0} \rightarrow K^{0 *} \bar{K}^{0}$ [ [4].

From the above analysis, we therefore see that the CP phase $\alpha$ can be cleanly extracted from measurements of the decays of $B_{d}^{0}$ and $B_{s}^{0}$ mesons to two different final states consisting of one neutral kaon (i.e. $K^{0}$ or any of its excited states) and one neutral anti-kaon (i.e. $\bar{K}^{0}$ or any excited state). Finally, we note that the $K^{*} \bar{K}^{*}$ final state consists of three helicity states. Each helicity state can be then considered a distinct final state for the purposes of our analysis. Thus, by applying our method to two different $K^{*} \bar{K}^{*}$ helicity states, $\alpha$ can be obtained from $B_{d, s}^{0} \rightarrow K^{*} \bar{K}^{*}$ decays alone.

The branching ratios of the pure pure $b \rightarrow d$ penguin decays $B_{d}^{0}(t) \rightarrow K^{(*)} \bar{K}^{(*)}$ are expected to be quite small, of order $10^{-6}$. Hence this method is ideally suited to hadron colliders as they produce an enormous number of $B$ mesons. Furthermore, in all cases, the kaon or anti-kaon can be detected using its decays to charged $\pi$ 's or $K$ 's only; this method does not require the detection of $\pi^{0}$ 's. Therefore hadron colliders will be able to use this technique to measure $\alpha$ - all that is required is good $\pi / K$ separation. And if $\pi^{0}$ 's can be detected, this simply increases the detection efficiency for the various final states.

\section{New Physics in $B_{d, s}^{0} \rightarrow K^{(*)} \bar{K}^{(*)}$}

The decays $B_{d, s}^{0} \rightarrow K^{(*)} \bar{K}^{(*)}$ are pure penguin decays and hence could be sensitive to newphysics effects. Consider the decays $B_{s} \rightarrow K^{(*)} \bar{K}^{(*)}$, which are dominated to a very good approximation by a single amplitude in the standard model (see Eq. 6). Hence a measurement of CP violation, such as a direct CP asymmetry, will be a clear sign of new physics in the $b \rightarrow s$ penguin.

New physics in the $b \rightarrow s$ penguin can also affect the more well known decay $B_{d} \rightarrow$ $\phi K_{S}$ [6]. However, note that the new-physics operator for $B_{s} \rightarrow K^{(*)} \bar{K}^{(*)}$ is of the form $O_{d}=\bar{d} \Gamma_{1} d \bar{s} \Gamma_{2} b$, where $\Gamma_{1,2}$ are some Lorentz operators, while for $B_{d} \rightarrow \phi K_{S}$ the new-physics operator is of the form $O_{s}=\bar{s} \Gamma_{1} s \bar{s} \Gamma_{2} b$. There are models of new physics where the operators $O_{s}$ and $O_{d}$ are related. For example, consider models with an additional vector-singlet charge $-1 / 3$ quark $h$ which mixes with the ordinary down-type quarks $d, s$ and $b$. (These models are generally motivated by $E_{6}$ grand unified theories.) This then generates flavour-changing effects through the $Z b \bar{s}$ FCNC coupling [7]. This coupling will then generate the operators $O_{s, d}$ but with the same strength.

Models of new physics which contain exotic fermions generally have additional neutral $Z^{\prime}$ gauge bosons. If the $s^{-}, b$ - and $h$-quarks have different quantum numbers under the new $U(1)$ symmetry, their mixing will also induce FCNC's due to $Z^{\prime}$ exchange [8, 9] which will 
then generate $O_{s, d}$, but again with the same strength. Hence in such models CP violation in $B_{d} \rightarrow \phi K_{S}$ and $B_{s} \rightarrow K^{(*)} \bar{K}^{(*)}$ will be correlated.

On the other hand, consider another model of new physics: R-parity breaking supersymmetry (SUSY). The most general superpotential of the MSSM with $S U(3) \times S U(2) \times U(1)$ gauge symmetry which breaks R-parity is

$$
\begin{aligned}
\mathcal{W}_{\not R} & =\frac{1}{2} \lambda_{[i j] k} L_{i} L_{j} E_{k}^{c}+\lambda_{i j k}^{\prime} L_{i} Q_{j} D_{k}^{c} \\
& +\frac{1}{2} \lambda_{i[j k]}^{\prime \prime} U_{i}^{c} D_{j}^{c} D_{k}^{c}+\mu_{i} L_{i} H_{2} .
\end{aligned}
$$

Here $L_{i}\left(Q_{i}\right)$ and $E_{i}\left(U_{i}, D_{i}\right)$ are the left-handed lepton (quark) doublet and lepton (quark) singlet chiral superfields, where $i, j, k$ are generation indices and $c$ denotes a charge-conjugate field. $H_{1,2}$ are the chiral superfields representing the two Higgs doublets. The non-observation of proton decay imposes very stringent conditions on the simultaneous presence of both the baryon-number and lepton-number violating terms in the Lagrangian [10].

The B-violating couplings $\lambda^{\prime \prime}$ are antisymmetric in the last two indices. Hence the operator $O_{s}$ cannot be generated at tree level and so there will no significant effects in $B_{d} \rightarrow \phi K_{S}$. On the other hand, the operator $O_{d}$ can be generated at tree level and hence can lead to CP violation in $B_{s} \rightarrow K^{(*)} \bar{K}^{(*)}$.

The $L$-violating couplings are given in terms of four-component Dirac spinors by [11]

$$
\begin{aligned}
\mathcal{L}_{\lambda^{\prime}}= & \lambda_{i j k}^{\prime}\left[\tilde{e}_{L}^{i} \bar{d}_{R}^{k} u_{L}^{j}+\tilde{u}_{L}^{j} \bar{d}_{R}^{k} e_{L}^{i}+\tilde{d}_{R}^{k *} \bar{e}_{L}^{i c} u_{L}^{j}\right. \\
& \left.-\tilde{\nu}_{L}^{i} \bar{d}_{R}^{k} d_{L}^{j}-\tilde{d}_{L}^{j} \bar{d}_{R}^{k} \nu_{L}^{i}-\left(\tilde{d}_{R}^{k}\right)^{*}\left(\bar{\nu}_{L}^{i}\right)^{c} d_{L}^{j}\right] \\
+ & \text { h.c. }
\end{aligned}
$$

In this case both operators $O_{s}$ and $O_{d}$ will be generated, but in general with different strengths. Thus, in this model CP violation in $B_{d} \rightarrow \phi K_{S}$ and $B_{s} \rightarrow K^{(*)} \bar{K}^{(*)}$ can be quite different.

Finally, we return to the measurement of the CP phase $\alpha$ via $B_{d, s}^{0} \rightarrow K^{(*)} \bar{K}^{(*)}$. If the value of $\alpha$ obtained via this method differs from that measured in $B_{d} \rightarrow \pi \pi$ or $B_{d} \rightarrow \rho \pi$, this will be evidence of new physics in the $b \rightarrow d$ or $b \rightarrow s$ penguin amplitudes.

\section{Conclusion}

In conclusion, we have a presented a new method to measure $\alpha$ using $B_{d, s}^{0} \rightarrow K^{(*)} \bar{K}^{(*)}$. Because these processes are pure penguin decays, they are particularly sensitive to new physics. We have described several ways of detecting new physics in such decays.

Acknowledgements: This work was financially supported by NSERC of Canada. 


\section{References}

[1] B. Aubert et al. [BABAR Collaboration], arXiv:hep-ex/0203007; K. Trabelsi [BELLE Collaboration], talk given at the XXXVII ${ }^{\text {th }}$ Rencontres de Moriond Electroweak Interactions and Unified Theories, 2002.

[2] M. Gronau and D. London, Phys. Rev. Lett. 65, 3381 (1990).

[3] A.E. Snyder and H.R. Quinn, Phys. Rev. D48, 2139 (93); H.R. Quinn and J.P. Silva, Phys. Rev. D62: 054002 (2000).

[4] A. Datta and D. London, Phys. Lett. 533B, 65 (2002).

[5] The fact that one needs one piece of theoretical input to obtain CP phase information from $b \rightarrow d$ penguin decays was noted in D. London, N. Sinha and R. Sinha, Phys. Rev. D60: 074020 (1999).

[6] Y. Grossman and M. P. Worah, Phys. Lett. B 395, 241 (1997) arXiv:hep-ph/9612269; D. London and A. Soni, Phys. Lett. B 407, 61 (1997) arXiv:hep-ph/9704277].

[7] Y. Nir and D. Silverman, Phys. Rev. D42, 1477 (1990).

[8] E. Nardi, Phys. Rev. D48, 1240 (1993); J. Bernabéu, E. Nardi and D. Tommasini, Nucl. Phys. B409, 69 (1993).

[9] K. Leroux and D. London, Phys. Lett. 526B, 97 (2002).

[10] I. Hinchliffe and T. Kaeding, Phys. Rev. D 47, 279 (1993); C. E. Carlson, P. Roy and M. Sher, Phys. Lett. B 357, 99 (1995) arXiv:hep-ph/9506328; A. Y. Smirnov and F. Vissani, Phys. Lett. B 380, 317 (1996) [arXiv:hep-ph/9601387].

[11] See for example A. Datta, J. M. Yang, B. L. Young and X. Zhang, Phys. Rev. D 56, 3107 (1997) arXiv:hep-ph/9704257. 